\title{
DEMOGRAFIE
}

\section{Altern gestalten}

\author{
Thomas Mäule
}

\begin{abstract}
Nicht der demografische Wandel an sich stellt die eigentliche Herausforderung dar, sondern die "demografische Ignoranz", wie es James W. Vaupel, Direktor des Rostocker Max-PlanckInstituts für demografische Forschung, genannt hat. Auch die Sozialwirtschaft muss sich endlich der neuen Situation stellen.
\end{abstract}

Durch den demografischen Wandel steht insbesondere die Altenhilfe vor ganz neuen Herausforderungen. Sie gewinnt nicht nur im quantitativen Sinne an Bedeutung. Auch das Spektrum weitet sich im Spannungsfeld zwischen Hilfebedürftigkeit auf der einen und der Förderung von Potenzialen auf der anderen Seite. Fünf Aufgabenbereiche werden im Folgenden skizziert und im Blick auf Altenhilfeträger konkretisiert.

\section{Aufgabe: Differenzierte Alters- bilder erkennen, kommunizieren und Angebote neu ausrichten}

Das Alter hat viele Gesichter. Ältere Menschen sind eine heterogene Gruppe. Altersfixierte Standardlösungen gibt es nicht. Je älter wir werden, umso größer sind die interindividuellen Unterschiede. Dies ist auch einer der Gründe, warum Wissenschaftler von einem gesetzlich definierten Renteneintrittsalter abraten. Konzepte, die auf homogene Bedürfnisse alter Menschen setzen, gehen an Bedarfen häufig vorbei. Das macht die Sache so kompliziert. Auf diese individuell sehr verschiedenen Bedürfnisse differenziert zu antworten, ist eine erste grundlegende Aufgabe.

Nicht gering zu schätzen dürfte die Selbstreflexion der eigenen Altersbilder sein. Wie werden Kompetenzen und Potenziale des Alters kommuniziert? Wie werden Krankheit, Sterben und Tod thematisiert? Wird ein Auto- matismus zwischen Alter und Pflegebedürftigkeit suggeriert? In der gesellschaftlichen Diskussion wird nicht selten ein Altersbild gemalt, das einseitig nur die Einschränkungen oder nur die Potenziale betont. Aus dieser Gesamtlage ein realistisches und was Hoffnung und Angst angeht ausbalanciertes Bild vom Alter und seiner Zukunft zu komponieren, ist ein erster Schritt. Darauf basierend sind Angebote (gegebenenfalls neu) auf Alternsformen und Lebensstile auszurichten. Auf der einen Seite gilt es, Konzepte auf die erhöhte Verletzlichkeit des Menschen im hohen Alter zu fokussieren, auf der anderen Seite den Menschen in seinen potenziellen Kompetenzen wahrzunehmen und anzusprechen.

Das Spektrum Sozialer Arbeit weitet sich. Damit stehen Altenhilfeträger selbst in einem Prozess der Neuorientierung und Positionierung ihrer Wirkungsfelder. Nur differenzierte Antworten werden den heterogenen Anforderungen und den sich weiter ausdifferenzierenden Arbeitsformen der Altenarbeit gerecht.

\section{Aufgabe: Gelegenheiten schaffen, damit Potenziale sich entfalten können}

Diese Differenzierung hat eine Vielzahl von Implikationen für den kulturellen Umgang mit dem Alter. Wie können Chancen und Entwicklungspotenziale im Alter entfaltet werden? Welche Voraussetzungen für Partizipation, Teilhabe und Engagement müssen gegeben sein? Welchen Beitrag können ältere Menschen leisten?

Gerade im nachberuflichen Bereich kann Freiwilligenarbeit ein wichtiger Sinngeber und unentbehrlicher Beitrag für das Gemeinwesen sein. Die Bereitschaft von älteren Menschen, sich zu engagieren, ist hoch. Neben dem klassischen Ehrenamt suchen ältere Men-

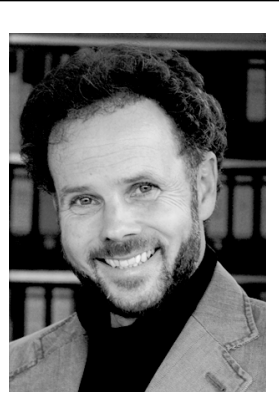

Dr. Thomas Mäule studierte Theologie, Sportwissenschaft, Gerontologie und Sozialmanagement. Er war Gemeindepfar-

rer, Studienleiter an der Evangelischen Akademie Bad Boll und Leiter des Grundsatzreferates im Diakonischen Werk Württemberg. Derzeit ist er Referent für Theologie und Ethik bei der Evangelischen Heimstiftung GmbH in Stuttgart. Die Organisation ist mit 6.800 Plätzen der größte Anbieter von Altenhilfedienstleistungen in Baden-Württemberg. In 61 Alten- und Pflegeheimen arbeiten rund 5.500 Beschäftigte.

E-Mail t.maeule@ev-heimstiftung.de

schen zunehmend neue Formen des Engagements mit mehr Selbstbestimmung. Einrichtungen müssen für freiwillig Engagierte beteiligungsfreundlicher werden. Sie dürfen die Freiwilligen nicht nur für die unliebsamen Arbeiten einsetzen, für die sich sonst niemand findet. Notwendig sind neue Räume für Mitbestimmung und Mitgestaltung, notwendig ist die Vernetzung mit Institutionen und Anlaufstellen im Gemeinwesen. Es geht darum, Potenziale von Menschen zu entdecken und zu aktivieren sowie alle Interessenten an einen Tisch zu holen. Erfahrungen zeigen, dass im Gemeinwesen ein riesiges Potenzial schlummert. Dies gilt es zu nutzen für Angebote der Begegnung, für Besuchsdienste, zur Unterstützung pflegender Angehöriger, für Altenseelsorge.

Passgenaue Angebote sind von daher erforderlich, die ausdrücklich die Interessen älterer Menschen ansprechen, die ihre Bereitschaft wecken und fördern, sich in der Gesellschaft aktiv zu betätigen. Vielen alten Menschen fällt wegen der allgemeinen Abwertung des Alters das Zutrauen in eigene Kompetenzen schwer. Manche müssen erst daran erinnert werden, über welche Erfahrungen und Potenziale sie verfügen. Manchen fehlt der Mut, etwas Neues zu beginnen und eigene Kräfte zu entfalten. Hier liegen die Ansatzpunkte. 
Eine realistische Einschätzung der »hauptamtlichen Potenziale« ist ebenso angesagt. Der demografische Wandel wird die Mitarbeiterschaft verändern. Wir werden ältere Mitarbeitende haben, längere Arbeitszeiten. Die Alterung und der gleichzeitige Rückgang der Erwerbstätigen bringen zusätzlich einen Fachkräftemangel mit sich. Die Forschung zeigt, dass Älterwerden keineswegs mit einem automatischen Abbau der Fähigkeiten verbunden ist. Ältere Mitarbeitende mögen mit Zeitdruck, Komplexität und Verantwortung zum Teil mehr Mühe haben. Wir wissen aber auch, dass sie beispielsweise Informationen zielgerichteter verarbeiten und besser zwischen Wesentlichem und Unwesentlichem unterscheiden können. »Ältere « und »Jüngere« unterscheiden sich in der Gesamtleistung nicht notwendigerweise. Nötig aber sind altersgerechte Arbeitsbedingungen. An das Personalmanagement stellt sich von daher ein Bündel lebenszyklusorientierter, aufeinander abgestimmter Maßnahmen: vom Wissenstransfer zwischen älteren und jüngeren Mitarbeitenden, Work-Life-Balance bis hin zur betrieblichen Gesundheitsförderung und attraktiven und finanzierbaren Modellen zum Übergang in den Ruhestand.

\section{Aufgabe: Soziale Teilhabe und Selbstbestimmung ermöglichen}

Selbstbestimmte Teilhabe zu ermöglichen und zu fördern, ist ein unhintergehbares Leitprinzip der Altenhilfe. Beteiligung, nicht Ausschluss, Inklusion, nicht Exklusion, lautet die Perspektive. Selbstbestimmung heißt, über seine Angelegenheiten, auch in den einfachsten Lebensvollzügen, entscheiden zu können, Wahlmöglichkeiten zu haben, als mitverantwortlich handelndes Subjekt angesprochen zu werden.

Oft ist Fantasie erforderlich, wenn zum Beispiel bei Demenzkranken die Kommunikationswege sich verschoben haben: weg von eher kognitiven, hin zu eher leiblichen Ausdrucksweisen. Untersuchungen zeigen, dass auch schwerstpflegebedürftige Personen, mit intellektuellen Beeinträchtigungen und schwer verstehbaren Verhaltensweisen, als selbstbestimmungsfähige Personen ansprechbar sind. Diese Menschen auf die ihnen eigentümlichen Verhaltensweisen anzusprechen, ist nicht nur ihnen ange-

messen, sondern auch ein Gebot der Menschenwürde.

In der konkreten Alltagsarbeit heißt dies, ein Höchstmaß an Selbstbestimmung zum Merkmal von Ergebnisqualität zu machen. Dabei sind Konflikte zwischen den Wünschen von Personen und Interessen von Institutionen vorprogrammiert. Die Qualität der Arbeit hängt entscheidend davon $\mathrm{ab}$, wie mit solchen Konflikten umgegangen wird. Wie wird Teilhabe ein-

\section{»Ein Höchstmaß an Selbstbestimmung muss ein Merkmal von Ergebnisqualität sein"}

geübt? Wie mit Beschwerden umgegangen? Wie das Verhältnis und die Zusammenarbeit zwischen Hauptund Ehrenamtlichen gestaltet?

Auf der Organisationsebene heißt selbstbestimmte Teilhabe auch ein Höchstmaß an Partizipation für Mitarbeitende auf allen Ebenen. Auf der ordnungspolitischen Ebene bedeutet Selbstbestimmung eher Subjektförderung und Persönliche Budgets als die Subventionierung von Anbietern. Auf der Ebene der Interessenvertretung müssen Altenhilfeträger darauf achten, dass sie nicht nur für ältere Menschen oder an deren Stelle sprechen, sondern möglichst mit ihnen und mit dem Ziel, sie zu befähigen, ihre Interessen eigenständig zu vertreten. Ihre Intention muss sein, Kräfte der Selbstverantwortung und Selbstgestaltung freizusetzen.

\section{Aufgabe: Menschen in Grenz- situationen begleiten und Voraus- setzungen für umfassende Pflege schaffen}

Schwerstkranke und sterbende Menschen zu begleiten und ihnen die notwendige, von ihnen gewünschte Betreuung zukommen zu lassen, ist ein weiterer wichtiger Beitrag. Die Abhängigkeit von der Hilfe anderer ist für viele Menschen eine Schreckensvorstellung. Es bedeutet, die Kontrolle über das eigene Leben zu verlieren und Bedingungen ausgeliefert zu sein, die man nicht selbst steuern kann.

Der Zustand der Abhängigkeit ist nicht per se eine schreckliche Erfah- rung. Sie wird es erst dann, wenn ich weder der Person noch der Institution, in deren Rahmen für mich gesorgt wird, vertrauen kann. Damit geraten die Person des Hilfeleistenden und die Unternehmenskultur in den Blick. Einrichtungsträger sind aufgefordert, ein umfassendes Verständnis von Pflege im Blick zu haben, die es den Pflegenden ermöglicht, die personale Dimension in der Beziehung zum Gepflegten auszugestalten. Mit der personalen Dimension wird die
Bedeutung der fachlichen Kompetenz nicht geschmälert. Nicht nur der Hilfeempfangende lässt sich auf eine vertrauensvolle Beziehung ein, sondern auch der Hilfegebende. Nicht zuletzt aus Angst vor Bindung und Ausgeliefertsein, Gefühlsdiffusion und Kontrollverlust schützen viele Pflegekräfte ihr Selbst und ziehen sich auf die technisch-korrekte Durchführung der Pflegehandlungen zurück. Doch ohne die eigene Person einzubringen, wird man auf Dauer nicht pflegen können.

Wichtig ist es, Räume zu schaffen, in denen Mitarbeitende ihre Erfahrungen und ihre Motivation reflektieren können, in denen aber auch Zweifel, Unsicherheiten und kritische Fragen ihren Platz haben.

Zur seelischen Bewältigung der Pflegebedürftigkeit gehört ebenso die psychologische und seelsorgliche Begleitung. Mit dem Wandel der Altersstruktur wandelt sich das Feld der Altenseelsorge vom Sonderfall zum Normalfall. Für Kirchengemeinden wird Seelsorge an alten Menschen, ob zu Hause oder in Einrichtungen, vom Nebenort zum Hauptort pastoralen Handelns.

Die großen ethischen Konfliktthemen wie die »aktive Sterbehilfe « machen deutlich, wie aktuell ethische Fragen in der Pflege sind und wie notwendig es für Einrichtungsträger ist, sich aktiv an dieser Diskussion zu beteiligen. Eine gute Palliativkultur und Abschiedskultur zu gestalten, ist eine weitere Aufgabe. Hier kommt es wesentlich auf die Verknüpfung von pflegerischem und medizinischem 
Wissen an, auf die Kooperation von Haupt- und Ehrenamtlichen, vor allem aber auf den Respekt vor der Individualität am Lebensende.

\section{Aufgabe: Das Miteinander der Generationen stärken}

Dass Fragen des Alters grundsätzlich in Generationen übergreifenden Kontexten zu behandeln sind, macht eine fünfte Herausforderung deutlich. Was muss konkret getan werden, damit die Solidarität zwischen den Generationen Wirklichkeit bleibt und Bestand hat? Wichtig ist, dass Solidarität nicht als Überforderung erfahren wird, sondern als persönlicher Gewinn - von allen Generationen in allen Lebensphasen.

Für den Fortbestand der Familiensolidarität dürften deutlich verbesserte Rahmenbedingungen entscheidend sein, um die Vierfachbelastung von Kindern, Bildung, Angehörigenpflege und Beruf auch tatsächlich vereinbaren zu können. Flexible Arbeitszeitmodelle, ein Angebot zur Kinderbetreuung in der Nähe des Arbeitsplatzes oder kostengünstiges Essen für Familienangehörige können erste Schritte sein.

Zur Entlastung der Familie - aber auch künftiger Generationen, die nicht mehr in gleichem Umfang auf Angehörige zurückgreifen können werden Tages-, Kurzzeitpflegeeinrichtungen, Koordinierungsstellen, an die sich pflegende Angehörige wenden können, immer wichtiger.

Außerhalb von Familie und Beruf besteht ein Bedarf an Projekten, die sich um eine Stärkung der generationenübergreifenden Solidarität bemühen, Das Projektbüro »Dialog der Generationen « in Berlin verfolgt das Anliegen, den Dialog zwischen Alt und Jung zu fördern (www.generationendialog.de/projektebuero.phb). Durch Austausch und Unterstützungsleistungen lernen Kinder und Ältere gegenseitiges Verständnis. Die Gemeinsamkeiten, so zeigen die Projekte, sind größer als angenommen. Seit dem Jahre 1982 propagieren die Vereinten Nationen das »Miteinander der Generationen «. Den Zusammenhalt der Gesellschaft zu stärken - darin liegt die eigentliche Zukunftsaufgabe.

\section{Expertenwissen für Führungskräfte}

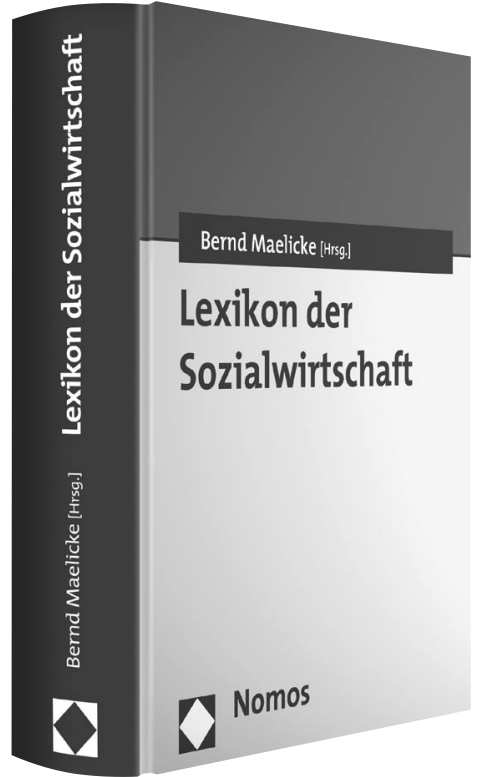

\section{Lexikon der Sozialwirtschaft}

Herausgegeben von Prof. Dr. Bernd Maelicke

2007, 1.128 S., geb., 98,-€,

ISBN 978-3-8329-2511-6

Das Werk umfasst ca. 700 Stichworte von über 130 Autoren aus den einschlägigen Fachdisziplinen Betriebswirtschaft, Volkswirtschaft, Sozialpolitik, New Public Management, Recht, Erziehungswissenschaften, Sozialarbeit/Sozialpädagogik und Psychologie und richtet sich an die Führungskräfte in der Sozialwirtschaft/Sozialmanagement, der Aus- und Weiterbildung sowie an die Wissenschaft.

"Es ist das erste Überblickswerk zu der Thematik und stellt auf $1.128 \mathrm{Sei}$ ten dar, was gegenwärtig fachlich und sachlich der Stand des Wissens zu sozialwirtschaftlichen Fragen ist."

Prof. Dr. Wolf Rainer Wendt, Zeitschrift für Betreuungsmanagement 69/o8

"Ein exzellentes Nachschlagewerk für den Bereich der Sozialen Arbeit und den angrenzenden Arbeitsfeldern...ein zu empfehlendes Nachschlagewerk für all jene, die fundierte Informationen schnell auffinden müssen und dabei einen wissenschaftlichen Anspruch haben."

Tarek Bamberger, www.sozialarbeitsnetz.de, November 07 